\title{
Medidas de protección alveolar en pacientes con COVID-19**
}

Alveolar protection measures in patients with COVID-19

Medidas de proteção alveolar em pacientes com COVID-19

Yessica García Salas, ${ }^{\star}$ Beatriz Cruz Muñoz, ${ }^{*}$ Fabricio Ortiz Larios, ${ }^{\star}$ José David Torres Adalid

\section{RESUMEN}

COVID-19, la enfermedad ocasionada por SARS-CoV-2, fue declarada pandemia por la OMS a principios de marzo 2020. En México, hasta noviembre 2020 habían sido confirmados más de 995,000 casos y cerca de 100,000 defunciones, situándose dentro de los 10 países con mayor tasa de infectados a nivel mundial. La gran mayoría de pacientes cursa con síntomas leves que no amerita hospitalización, pero los pacientes con cuadros severos de insuficiencia respiratoria pueden requerir ventilación mecánica invasiva, tratamiento que en México se ha asociado a una mortalidad de hasta $73.7 \%$.

La ventilación mecánica es una terapia que puede salvar vidas, pero no está exenta de riesgos y una programación inadecuada también puede ocasionar daño por diversos mecanismos denominados en conjunto VILI (Ventilatorinduced lung injury). Más de 50 años de búsqueda han dictado las directrices para el manejo óptimo de la ventilación mecánica, pero esta nueva pandemia ha abierto el panorama a nuevas investigaciones. La ventilación protectora pulmonar: VT de 6-8 $\mathrm{mL} / \mathrm{kg}$, presión meseta $<30 \mathrm{cmH}_{2} \mathrm{O}$, ha mejorado los desenlaces clínicos en pacientes con y sin SDRA en los últimos 20 años, aunque el COVID-19 tiene particularidades la evidencia sugiere otorgar la misma estrategia.

Palabras clave: Ventilación mecánica, protección alveolar, COVID-19.

\section{ABSTRACT}

COVID-19, the disease spectrum caused by the new beta coronavirus SARSCoV-2 was declared as a pandemic by the WHO in March 2020. Until November 2020 more than 995,000 cases and nearly 100,000 deaths had been confirmed have been confirmed in Mexico, being one of the 10 countries with the highest index of cases. Most patients have a mild disease not requiring hospitalization, but severe cases with respiratory failure may require mechanical ventilation with a high mortality rate up to $73.7 \%$ in some series in Mexico. Mechanical ventilation saves lives but, it is not out of risk as it may cause harm through many mechanisms named VILI on the whole (ventilator-induced lung injury). After more than 50 years since its first description, protective mechanical ventilation has become the standard of care in ARDS patients, but this pandemic has opened the gap for new research. Protective mechanical ventilation: Iow tidal volumes 6-8 $\mathrm{mL} / \mathrm{kg}$ predicted body weight (PBW) and plateau pressure < $30 \mathrm{cmH}_{2} \mathrm{O}$ has improved patients' outcomes in the last 20 years. Even though COVID-19 has certain differences with the "classical» ARDS, evidence-based medicine suggests the same strategies should be followed.

Keywords: Mechanical ventilation, alveolar protection, COVID-19.

\section{RESUMO}

COVID-19, a doença causada pelo SARS-CoV-2, foi declarada pandemia pela OMS no início de março de 2020. No México, até novembro de 2020 foram confirmados mais de 995,000 casos e quase 100,000 mortes, classificando-se em 10 países com a maior taxa de infectados em todo o mundo. A grande maioria dos pacientes apresenta sintomas leves que não justificam a hospitalização, mas os pacientes com insuficiência respiratória grave podem requerer ventilação mecânica invasiva, um tratamento que no México foi associado a uma taxa de mortalidade de até $73.7 \%$. A ventilação mecânica é uma terapia que salva vidas, mas não é isenta de riscos, e uma programação inadequada também pode causar danos por vários mecanismos chamados coletivamente de VILI (Ventilatorinduced lung injury). Mais de 50 anos de pesquisa ditaram as diretrizes para $O$

** Primer lugar del Concurso «Tazón Alvéolo 2020».

* Hospital Civil de Guadalajara «Fray Antonio Alcalde».

Recibido: 13/11/2020. Aceptado: 22/12/2020

Citar como: García SY, Cruz MB, Ortiz LF, Torres AJD. Medidas de protección alveolar en pacientes con COVID-19. Med Crit. 2020;34(6):341-348. https://dx.doi. org/10.35366/98164

www.medigraphic.com/medicinacritica gerenciamento ideal da ventilação mecânica, mas esta nova pandemia abriu o cenário para novas pesquisas. Ventilação protetora pulmonar: VT de $6-8 \mathrm{~mL} / \mathrm{kg}$, pressão de platô $<30 \mathrm{cmH}_{2} \mathrm{O}$, melhorou os desfechos clínicos em pacientes com e sem SDRA nos últimos 20 anos, embora COVID-19 tenha particularidades, as evidências sugerem conceder as mesmas estratégia.

Palavras-chave: Ventilação mecânica, proteção alveolar, COVID-19.

\section{INTRODUCCIÓN}

Desde su primera descripción en 1967 por Ashbaugh y colaboradores, el síndrome de distrés respiratorio agudo (SDRA) ha sido un desafío para los médicos especializados en Cuidados Intensivos. Más de 50 años de búsqueda han generado una gran cantidad de evidencia científica que guiaron la búsqueda de mejores estrategias de tratamiento y un manejo óptimo de la ventilación mecánica. Sin embargo, una nueva pandemia puso en tela de juicio este conocimiento y ha abierto el panorama a nuevas investigaciones.

COVID-19 es la enfermedad ocasionada por SARSCoV-2, fue declarada pandemia por la Organización Mundial de la Salud a principios de marzo 2020. Desde sus primeros reportes en diciembre 2019 en Wuhan, China, fue descrita su asociación con insuficiencia respiratoria aguda hipoxémica, con una proporción mayor a la ocasionada por SARS-CoV (2002-2004) y MERS (2012), otros síndromes también ocasionados por coronavirus. ${ }^{1}$

Hasta la primera mitad de noviembre 2020, el número de casos confirmados a nivel mundial superaba los 51 millones y ha ocasionado más de un millón de fallecimientos. En México, el primer caso documentado se presentó el 27 de febrero de $2020,{ }^{2}$ y hasta noviembre 2020 habían sido confirmados más de 995,000 casos y cerca de 100,000 defunciones, situándonos dentro de los 10 países con mayor tasa de infectados y letalidad a nivel mundial. ${ }^{3}$

\section{SARS-CoV-2 Y COVID-19}

SARS-CoV-2 es un beta coronavirus que guarda relación estrecha con subtipos aislados en murciélagos, lo cual indica su posible origen zoonótico. Se compone de ácido ribonucleico monocatenario (RNA), ${ }^{3}$ se une al epitelio respiratorio a través de la proteína $S$ de su superficie y ocasiona una enfermedad respiratoria aguda que 
se transmite principalmente a través de secreciones del tracto respiratorio. ${ }^{2}$

Las manifestaciones clínicas van desde síntomas leves hasta cuadros graves con insuficiencia respiratoria, con una gran proporción de asintomáticos. Los síntomas más comunes son fiebre $(83 \%)$, tos $(82 \%)$ y dificultad respiratoria (31\%). Síntomas gastrointestinales como vómito, diarrea y dolor abdominal son descritos en 2 al $10 \%$ de los casos y generalmente preceden a la fiebre y los síntomas respiratorios. ${ }^{4}$

La gran mayoría de casos con síntomas leves no ameritan hospitalización, pero quienes presentan cuadros severos con insuficiencia respiratoria hipoxémica pueden requerir atención en Unidades de Cuidados Intensivos y Ventilación Mecánica Invasiva, estrategia de tratamiento que en México se ha asociado a una mortalidad de hasta $73.7 \% .^{5}$

\section{LESIÓN PULMONAR INDUCIDA POR EL VENTILADOR}

La ventilación mecánica es una terapia que en sus más de siete décadas de uso ha demostrado salvar vidas; sin embargo, no está exenta de riesgos y una programación inadecuada también puede ocasionar daño. En la actualidad, se reconocen diversos mecanismos por los cuales la ventilación mecánica ocasiona lesión pulmonar o VILI (Ventilator-induced lung injury) estos son: volutrauma, barotrauma, atelectrauma y biotrauma, se describen en la Tabla 1.

Gracias a la evidencia científica que se ha acumulado a lo largo del tiempo, se han modificado las estrategias de administración de este soporte, mejorando los resultados clínicos, principalmente en aquéllos que padecen SDRA, hasta llegar a lo que hoy se conoce como ventilación protectora pulmonar que consiste en administrar volúmenes corrientes bajos y una presión positiva al final de la espiración (PEEP) para mantener las unidades alveolares reclutadas, teniendo como límite una presión meseta $<30 \mathrm{cmH}_{2} \mathrm{O}$.

Hoy en día, la ventilación protectora pulmonar se ha convertido en el estándar de manejo para los sujetos con SDRA, pero no sólo este grupo de casos se bene-

Tabla 1: Mecanismos de lesión pulmonar inducida por el ventilador.

\begin{tabular}{|c|c|}
\hline Barotrauma & $\begin{array}{l}\text { Lesión pulmonar por sobredistensión alveolar, ocasiona- } \\
\text { da por volumen tidal excesivo }\end{array}$ \\
\hline Barotrauma & $\begin{array}{l}\text { Lesión pulmonar causada por una alta presión transpul- } \\
\text { monar que rompe las estructuras alveolares }\end{array}$ \\
\hline Atelectrauma & $\begin{array}{l}\text { Lesión pulmonar ocasionada por la apertura y cierre } \\
\text { cíclico de unidades alveolares }\end{array}$ \\
\hline Biotrauma & $\begin{array}{l}\text { Lesión pulmonar ocasionada por mediadores inflama- } \\
\text { torios, que además de lesión local pueden ocasionar } \\
\text { inflación sistémica y falla orgánica múltiple }\end{array}$ \\
\hline
\end{tabular}

fician de dicha estrategia, personas con otras causas de falla respiratoria hipoxémica, incluso aquéllos con mecánica pulmonar normal, presentan menos complicaciones, mejorando los resultados clínicos a corto y largo plazo. ${ }^{6}$

\section{HETEROGENEIDAD DE LA PRESENTACIÓN CLÍNICA DEL COVID-19 GRAVE}

Aunque la mayoría de infectados por SARS-COV-2 que requieren ventilación mecánica invasiva por insuficiencia respiratoria aguda hipoxémica cumplen con la definición de Berlín para SDRA ${ }^{7}$ (Tabla 2), no todos presentan la forma típica de la enfermedad.

Gattinoni y colaboradores en tres diferentes reportes describen casos de hipoxemia severa con una fracción de corto circuito elevada en el contexto de una distensibilidad relativamente normal, atribuyendo como principal mecanismo de hipoxemia la alteración de la coeficiente ventilación/perfusión. Propone dos fenotipos primarios: el tipo L (Low) caracterizado por bajos niveles de elastancia pulmonar, bajo peso pulmonar y por ende baja capacidad de reclutamiento; y el tipo $\mathrm{H}$ (High) con valores altos de elastancia, mayor peso pulmonar y por tanto mayor capacidad de reclutamiento, siendo este último el más parecido a la descripción de un SDRA grave típico. Refieren que la mayoría de los casos se presentan de manera temprana con fenotipo $L$ y algunos avanzan a fenotipo $\mathrm{H}$, con un empeoramiento de la lesión pulmonar inicial, pudiendo atribuirse al curso natural de la enfermedad y/o la lesión pulmonar autoinducida. $^{8}$

Sólo un tercio de los sujetos analizados por Gattinoni y su equipo corresponden al fenotipo $\mathrm{H}$, datos que son contradictorios a otras series reportadas. En Nueva York, Schenck y colaboradores ${ }^{9}$ reportaron 257 pacientes con una distensibilidad estática de $27 \mathrm{~cm} / \mathrm{mL} / \mathrm{H}_{2} \mathrm{O}$ y $\mathrm{PaO}_{2} / \mathrm{FiO}_{2}$ promedio de 129. Cummings y su grupo de investigadores ${ }^{10}$ encontraron en 267 pacientes una distensibilidad estática de $25 \mathrm{~cm} / \mathrm{mL} / \mathrm{H}_{2} \mathrm{O}$ con $\mathrm{PaO}_{2} / \mathrm{FiO}_{2}$ de 103. Si bien estos datos parecen contradictorios, existe la posibilidad de que COVID-19 tenga diferentes fenotipos de SDRA, lo que explicaría la gran diversidad de presentación clínica. Sabemos que el SDRA es un síndrome por excelencia heterogéneo, hasta dentro de un mismo individuo, donde diferentes zonas del parénquima pulmonar pueden tener características macro y microscópicas y una mecánica ventilatoria distintas.

La heterogeneidad del SDRA y el intentar un manejo personalizado no son conceptos nuevos, desde hace dos décadas se ha intentado clasificar a los pacientes con SDRA en subgrupos de acuerdo con sus características clínicas, biológicas, respuesta al tratamiento y desenlaces clínicos. El análisis de dos grandes ensayos clínicos pertenecientes al grupo ARDSnet (ARMA 
Tabla 2: Criterios de diagnóstico y clasificación del SDRA, Consenso de Berlín 2012.

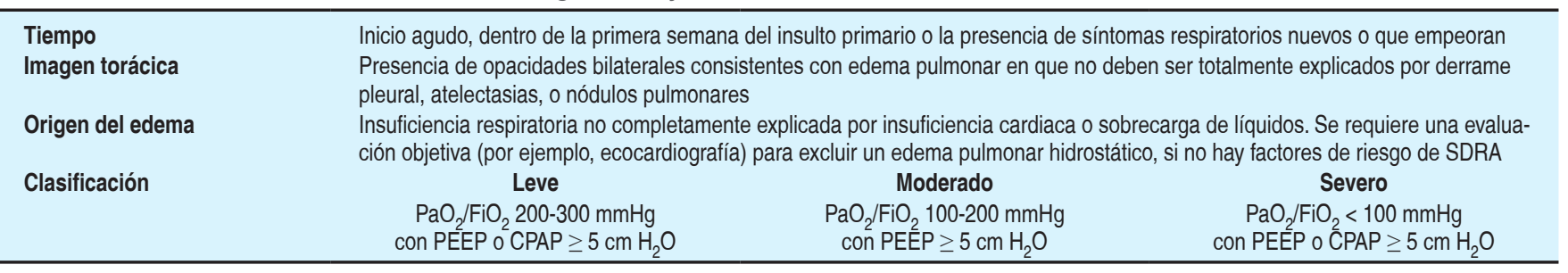

$\mathrm{SDRA}=$ síndrome de distrés respiratorio agudo, $\mathrm{PaO}_{2}=$ presión arterial de oxígeno, $\mathrm{FiO}_{2}=$ fracción inspirada de oxígeno, PEEP = presión positiva al final de la espiración, $\mathrm{CPAP}=$ presión positiva continua de la vía aérea .

y ALVEOLI) usando el modelo de clases latentes identificó dos fenotipos del SDRA, el tipo hiperinflamatorio que se beneficia de una ventilación con niveles altos de PEEP y una estrategia conservadora de líquidos parenterales; $y$ fenotipo hipoinflamatorio que tiene una mejor respuesta a bajos niveles de PEEP, ${ }^{11}$ estos conceptos han vuelto a ser puestos sobre la mesa, con base en las observaciones de pacientes con COVID-19 grave.

\section{VENTILACIÓN MECÁNICA PROTECTORA PULMONAR}

Dada la importancia de la estrategia de ventilación protectora en casos con falla respiratoria aguda con y sin SDRA, abordaremos este enfoque de tratamiento integrado a la información sobre las particularidades encontradas en pacientes con COVID-19.

\section{Volumen tidal y presión meseta}

De forma inicial, se deberá medir la altura de los pacientes para ajustar el volumen tidal (VT) al peso predicho; esto es fundamental, ya que el peso predicho puede diferir notablemente del peso real del paciente, éste se calcula con la siguiente fórmula:

$$
\begin{aligned}
& \text { Hombres }=50+0.91(\text { altura }[\mathrm{cm}]-152.4) \\
& \text { Mujeres }=45.5+0.91(\text { altura }[\mathrm{cm}]-152.4)
\end{aligned}
$$

Se sugiere programar un VT entre $6-8 \mathrm{~mL} / \mathrm{kg}$ de peso predicho, fuerte recomendación basada en la evidencia encontrada por el ensayo clínico «ARMA» publicado en el año 2000. ${ }^{12}$ Dicho estudio comparó una estrategia de protección pulmonar utilizando VT bajo de 6-8 mL/ $\mathrm{kg}$ de peso predicho con el objetivo de mantener una presión meseta entre $25-30 \mathrm{cmH}_{2} \mathrm{O}$ contra VT de 10$12 \mathrm{~mL} / \mathrm{kg}$ de peso predicho y presiones meseta entre 45-50 $\mathrm{mH}_{2} \mathrm{O}$ (hasta ese momento la terapia habitual). La presión meseta es medida en una pausa de $0.5 \mathrm{se}$ gundos, con flujo cero y sin esfuerzo respiratorio, con el objetivo de evitar sesgos de medición.

En ese estudio, el VT promedio en los días uno a tres fue de $6.2 \mathrm{~mL} / \mathrm{kg}$ vs $11.8 \mathrm{~mL} / \mathrm{kg}$ de peso predicho, con presiones meseta promedio de $26 \mathrm{mH}_{2} \mathrm{O}$ vs $38 \mathrm{mH}_{2} \mathrm{O}$.
El ensayo fue detenido prematuramente cuando el grupo de bajo volumen tidal demostró una disminución significativa en la mortalidad (31 vs $40 \%$ ), con un mayor número de días libres de ventilación (12 vs 10 días) en comparación con la estrategia de ventilación tradicional. Esto se tradujo en una reducción del riesgo absoluto (RR) de $9 \%$, con un número necesario a tratar (NNT) de 11 pacientes. A partir de estos resultados, ventilar a los casos con VT de 6-8 mL/kg de peso predicho con un límite de presión meseta $<30 \mathrm{cmH}_{2} \mathrm{O}$ se convirtió en el estándar de manejo.

Estudios posteriores han reafirmado los hallazgos del estudio "ARMA» incluso en pacientes sin SDRA, ventilar a los pacientes con VT elevados puede ser perjudicial. ${ }^{13}$ El grupo de trabajo «IMPROVE» observó que sujetos sin patología pulmonar intubados únicamente para someterse a cirugía abdominal ventilados con VT de 6 a $8 \mathrm{ml} / \mathrm{kg}$ de peso predicho durante la cirugía tuvieron menor incidencia de problemas respiratorios, neumonía y menos días de estancia hospitalaria que aquéllos ventilados con volúmenes corrientes mayores. ${ }^{14} \mathrm{En}$ el año 2012, Serpa y colaboradores ${ }^{6}$ realizaron un metaanálisis sobre el uso de VT bajos y los resultados clínicos en casos sin SDRA, demostrando un menor daño pulmonar en pacientes ventilados con esta estrategia (RR 0.33 [0.23-0.47]; NNT 11), así como menor mortalidad (RR 0.64 [0.46-0.89]; NNT 23) y una estancia intrahospitalaria más corta.

En pacientes con COVID-19, diversos autores han sugerido utilizar una estrategia más liberal de ventilación en aquéllos con fenotipo L, llevando a volúmenes tidales $>8 \mathrm{~mL} / \mathrm{kg}$ de peso predicho (volúmenes intermedios entre $8-10 \mathrm{~mL} / \mathrm{kg}$ ), afirmando que este grupo, al tener una distensibilidad respetada, generan un menor strain dinámico y por tanto menor riesgo de generar lesión pulmonar inducida por el ventilador. ${ }^{8}$ Resulta difícil discernir si el beneficio de la ventilación protectora pulmonar puede ser atribuido únicamente al cuidado de la presión meseta o a la limitación del VT entre 6-8 mL/kg de peso predicho, o a la interacción de ambas, por tanto, debemos tener en cuenta el peso de la evidencia que sugiere los beneficios obtenidos por esta estrategia aún en pacientes sin SDRA antes de decidir cambiar este enfoque, que ha demostrado por décadas mejorar los resultados. 


\section{TITULACIÓN DE LA PRESIÓN POSITIVA} AL FINAL DE LA ESPIRACIÓN

Para mantener las unidades alveolares reclutadas utilizando volúmenes corriente bajos se requiere de la aplicación de presión positiva al final de la espiración (PEEP). La cantidad ideal de PEEP es aquella que mantiene las unidades alveolares reclutadas sin ocasionar su sobredistensión, siendo variable de un paciente a otro, incluso en el mismo en diferentes etapas de su evolución. Con un adecuado nivel de PEEP se pretende mantener al pulmón reclutado o «abierto».

Hablar de pulmón abierto es hacer referencia a un concepto teórico, donde se pretende mantener a las unidades alveolares pulmonares reclutadas por diferentes métodos, principalmente con niveles de PEEP por encima de la presión de cierre de los alveolos, al mismo tiempo que se mantiene un intercambio de gases óptimo, manteniendo una amplitud de presión lo más pequeña posible para optimizar la eliminación de dióxido de carbono. Existen múltiples métodos para titular la PEEP, sin hasta el momento haber encontrado la forma ideal de hacerlo, siendo este un tema controversial incluso antes de la pandemia por COVID-19.

El estudio ALVEOLI ${ }^{15}$ publicado en 2004 comparó las dos tablas ARDSnet (Tablas 3 y 4) para la titulación de PEEP: una de PEEP bajo contra PEEP alto en relación al porcentaje de $\mathrm{FiO}_{2}$ que requerían los pacientes para mantener metas de oxigenación, en conjunto con VT $6 \mathrm{~mL} / \mathrm{kg}$ y presión $<30 \mathrm{cmH}_{2} \mathrm{O}$. El ensayo fue detenido prematuramente por futilidad, sin encontrar diferencias en mortalidad, días de ventilación mecánica, barotrauma o fallas orgánicas. Aunque este ensayo tuvo varias limitaciones, no demostró que una estrategia fuese mejor que la otra, cabe señalar que el uso de PEEP alto fue asociado a mejores niveles de $\mathrm{PaO}_{2} / \mathrm{FiO}_{2}$ y distensibilidad pulmonar.

En el año 2008, Mercat y su equipo ${ }^{16}$ publicaron el estudio «Express» en el que compararon el uso de una estrategia de mínima distensión (PEEP 5-9 $\mathrm{cmH}_{2} \mathrm{O}$ ) contra una estrategia de reclutamiento incrementado (máximo PEEP para alcanzar una presión meseta entre 28-30 $\mathrm{cmH}_{2} \mathrm{O}$ ). No hubo diferencias en términos de mortalidad; sin embargo, el grupo de reclutamiento incrementado presentó más días libres de ventilación mecánica, mejor distensibilidad pulmonar y oxigenación.

Otra forma de titular la PEEP y mantener el pulmón abierto es mediante la maniobra denominada Decremental PEEP, en donde con el paciente sedado y sin esfuerzo respiratorio espontáneo, en modalidad controlada por presión, manteniendo una presión de distensión (Driving pressure) de $15 \mathrm{cmH}_{2} \mathrm{O}$, se realiza una maniobra de reclutamiento activo con altos niveles de PEEP 25-30 $\mathrm{cmH}_{2} \mathrm{O}$ por dos a tres minutos, posteriormente cada dos minutos se va disminuyendo $2 \mathrm{cmH}_{2} \mathrm{O}$ la PEEP, se hace el registro de la distensibilidad pulmonar y la saturación de oxígeno, se escoge el nivel de PEEP donde se obtienen los mejores resultados de estas variables.

Esta estrategia fue evaluada en el ensayo piloto «OLA» en 2016, ${ }^{17}$ donde comparado contra la tabla de PEEP bajo de ARDSnet, muestra resultados similares en mortalidad, días de ventilación mecánica e incidencia de barotrauma, dicho ensayo tuvo la limitación de tener un pequeño tamaño de muestra. Por ello, en el año 2017 esta maniobra fue nuevamente evaluada en el ensayo clínico $\mathrm{ART}^{18}$ el cual reclutó más de 1,000 pacientes, aquí observaron que el grupo en donde se aplicó decremental PEEP tuvo menos días de ventilación mecánica, pero también mayor mortalidad, mayor riesgo de barotrauma y mayor incidencia de neumotórax. Una crítica fuerte a este estudio es que durante la maniobra de reclutamiento inicial elevaron la PEEP hasta $35 \mathrm{cmH}_{2} \mathrm{O}$, un nivel tan elevado aumenta el riesgo de barotrauma y colapso hemodinámico, siendo una variable que pudo haber contribuido en sus resultados.

La titulación de la PEEP con base en la presión transpulmonar medida a través de un balón esofágico también ha sido analizada. En 2008 Talmor y colaboradores ${ }^{19}$ publicaron un ensayo piloto en el que demostraron que su utilización mejoraba los niveles de oxigenación y la distensibilidad pulmonar comparado con el cuidado estándar. En el $2019^{20}$ se realizó un estudio con mayor tamaño de muestra en el que se evaluó la titulación de PEEP mediante está técnica, sin demostrar que su uso fuera superior en términos de mortalidad, días de

Tabla 3: PEEP baja.

\begin{tabular}{lcccccccccccccc}
\hline $\mathrm{FiO}_{2}$ & 0.3 & 0.4 & 0.4 & 0.5 & 0.5 & 0.6 & 0.7 & 0.7 & 0.7 & 0.8 & 0.9 & 0.9 & 0.9 & 1.0 \\
$\mathrm{PEEP}$ & 5 & 5 & 8 & 8 & 10 & 10 & 10 & 12 & 14 & 14 & 14 & 16 & 18 & $18-24$ \\
\hline
\end{tabular}

$\mathrm{FiO}_{2}=$ fracción inspirada de oxígeno, $\mathrm{PEEP}=$ presión positiva al final de la espiración.

Tabla 4: PEEP alta.

\begin{tabular}{lcccccccccccccc}
\hline $\mathrm{FiO}_{2}$ & 0.3 & 0.3 & 0.3 & 0.3 & 0.3 & 0.4 & 0.4 & 0.5 & 0.5 & $0.5-0.8$ & 0.8 & 0.9 & 1.0 & 1.0 \\
$\mathrm{PEEP}$ & 5 & 8 & 10 & 12 & 14 & 14 & 16 & 16 & 18 & 20 & 22 & 22 & 22 & 24 \\
\hline
\end{tabular}

$\mathrm{FiO}_{2}=$ fracción inspirada de oxígeno, $\mathrm{PEEP}$ = presión positiva al final de la espiración. 
ventilación y complicaciones a la titulación empírica de PEEP, dicho estudio tuvo críticas por el hecho de ajustar la PEEP en el grupo control con tablas distintas a las del grupo ARDSnet, donde se utilizaban niveles más elevados de $\mathrm{PEEP}$ con relación a la $\mathrm{FiO}_{2}$.

Pese a todas estas maneras de titular la PEEP, hoy en día no se recomienda una sobre otra, las tablas de $\mathrm{PEEP} / \mathrm{FiO}_{2}$ del grupo de trabajo ARDSnet son prácticas y seguras, tienen además el objetivo de alcanzar una adecuada oxigenación, por tanto, son ampliamente recomendadas.

La campaña «sobreviviendo a la sepsis» y algunos Institutos Nacionales Estadounidenses recomiendan adoptar una estrategia de PEEP alto vs PEEP bajo en pacientes con SDRA secundario a COVID-19;21,22 $\sin$ embargo, no hay evidencia suficiente para soportar dicha recomendación. ${ }^{8}$

En COVID-19, Gattinoni y colaboradores ${ }^{8}$ sugieren $^{2}$ que los pacientes con fenotipo $L$ se beneficiarán de una estrategia de PEEP bajo, ya que a pesar de cursar con hipoxemia severa cuentan con una distensibilidad pulmonar preservada, los estudios tomográficos muestran poco tejido pulmonar afectado, con predominio de lesiones en vidrio deslustrado más que verdaderas zonas de consolidación, el peso pulmonar es bajo y, por tanto, existen pocas áreas que se podrían reclutar con una PEEP alta, inclusive podría condicionar mayor riesgo de sobredistensión y efectos perjudiciales sobre la hemodinamia.

Se cree que estos casos, al tener una alteración de la vasoconstricción pulmonar por hipoxia, la aplicación innecesariamente elevada de PEEP puede ocasionar una presión alveolar mayor a la presión del capilar pulmonar, empeorando la relación ventilación/perfusión y agravando la hipoxia. Sugieren mantener la PEEP en rangos entre 8-10 $\mathrm{cmH}_{2} \mathrm{O}$ en fenotipos $\mathrm{L}$. En el fenotipo $\mathrm{H}$, el cual posee baja distensibilidad, mayor peso pulmonar y cuenta con zonas más extensas de consolidación pulmonar tiene mayor potencial de reclutamiento y son quienes podrían verse beneficiados de PEEP más elevadas. $^{8}$

Debido a la respuesta tan variable que se puede presentar ante distintos niveles de PEEP, no se recomienda la configuración predeterminada. EI SDRA es heterogéneo hasta en un mismo individuo y esto condiciona que tanto la PEEP como el VT tengan un impacto distinto en las unidades alveolares de diferentes zonas del pulmón, lo cual dependerá del grado de compromiso funcional y/o anatómico que presente cada una de ellas.

La PEEP posee un efecto dual, puede ser benéfica al reclutar unidades pulmonares colapsadas, mejorando el intercambio de gases y minimizando el atelectrauma, pero también puede ser perjudicial al generar sobredistensión, empeorando la relación ventilación/perfusión e inducir inestabilidad hemodinámica. Por tanto, encontrar la PEEP óptima puede no depender de una sola variable y es difícil recomendar a un grupo de pacientes específicos una PEEP fija, pues ésta puede variar entre individuos. Muchos autores abogan por valorar al pie de la cama el potencial de reclutabilidad que poseen los sujetos con COVID-19 y así otorgar una configuración individualizada de PEEP, respetando la estrategia de ventilación protectora pulmonar. ${ }^{23}$

\section{POTENCIAL DE RECLUTABILIDAD}

Existen estrategias que permiten evaluar el potencial de reclutabilidad pulmonar, no han sido analizadas en grandes ensayos clínicos aleatorizados; sin embargo, han sido evaluados en estudios experimentales, así como series de casos y tienen el potencial de ser realizadas a la cabecera del paciente.

Uno de estos métodos es evaluar el cambio de los infiltrados pulmonares mediante tomografía computarizada ante distintos niveles de PEEP; esto es poco práctico, sobre todo en las condiciones actuales de la pandemia por COVID-19, donde el exceso de casos y el riesgo de contaminación de los espacios hace difícil el traslado al tomógrafo.

En donde un aumento progresivo en la pendiente de la curva indica el reclutamiento de unidades alveolares durante la inspiración. Existe colapso cuando el IS $<1$ (pendiente de forma cóncava) o sobredistensión cuando el IS > 1 (Pendiente de forma convexa). Una curva presión-tiempo lineal indica un cambio mínimo durante la inspiración $(I S=1)$, esto sugiere que no existe ni reclutamiento ni sobredistensión, existe un equilibrio de fuerzas.

Otras herramientas como la medición de la presión transpulmonar mediante un balón esofágico, el uso de tomografía por bioimpedancia eléctrica, funciones adaptadas a algunos ventiladores mecánicos como P/V Tool de Hamilton o PEEP-view de Engström permiten estimar esta capacidad de reclutamiento, son de gran utilidad, pero no están disponibles en todos los centros de atención. Por ello, algunos autores han propuesto el índice reclutamiento-inflación, el cual calcula el cambio en el volumen espiratorio ante cada cambio de PEEP, valores $>0.5$ con respecto a la inflación pulmonar basal sugieren mayor potencial de reclutamiento, una herramienta que puede realizarse al pie de cama, está ampliamente disponible, permitiendo una mejor programación de PEEP. ${ }^{24}$

En 2014 Chiumello y su grupo ${ }^{25}$ evaluaron la capacidad de reclutamiento pulmonar mediante tomografía y compararon los métodos más frecuentemente utilizados para la titulación de PEEP como son: 1) El método del estudio EXPRESS, la PEEP necesaria para alcanzar una presión meseta entre $28-30 \mathrm{cmH}_{2} \mathrm{O}$ con un VT de 
$6 \mathrm{~mL} / \mathrm{kg}$ de peso. 2) Análisis de curvas, en búsqueda de un índice de estrés = 1. 3) Estimación de la presión transpulmonar mediante balón esofágico con el objetivo de mantener esta presión entre $0-5 \mathrm{cmH}_{2} \mathrm{O}$ al final de la espiración. 4) La tabla ARDSnet de PEEP alta con relación al $\mathrm{FiO}_{2}$. Encontraron que los niveles de PEEP seleccionados por los métodos EXPRESS, índice de estrés y presiones esofágicas absolutas, no se relacionaron con la capacidad de reclutamiento pulmonar, mientras que los niveles de PEEP seleccionados por la tabla de $\mathrm{PEEP} / \mathrm{FiO}_{2}$ alta mostró una relación débil con esta capacidad de reclutamiento pulmonar $\left(r^{2}=0.29\right.$; $p$ $<0.0001)$. Cuando se clasificó la severidad de acuerdo con los criterios de Berlín, los métodos Express, índice de estrés y presión esofágica ofrecieron valores similares de PEEP independientemente de la severidad del SDRA. La tabla ARDSnet PEEP alto fue el único método que proporcionó niveles de PEEP más bajos en SDRA leve a moderado y PEEP más altas en SDRA severo ( $8 \pm 2$ y $11 \pm 3 \mathrm{~cm} \mathrm{H}_{2} \mathrm{O}$ frente a $15 \pm 3 \mathrm{~cm} \mathrm{H}_{2} \mathrm{O}$; $\mathrm{p}<0.05)$.

En el SDRA «clásico» el grado de severidad se relaciona con la extensión de la lesión pulmonar y, por ende, a un mayor potencial de reclutamiento; sin embargo, en el COVID-19, las alteraciones vasculares y de la relación ventilación/perfusión tienen un peso preponderante en la severidad de la hipoxemia que no podemos asumir que esté correlacionado con potencial de reclutamiento. Es preocupante que se exponga a un pulmón con pobre potencial de reclutamiento a niveles altos de PEEP, si este presenta una mecánica preservada, a pesar de mantener presiones meseta por debajo de $30 \mathrm{cmH}_{2} \mathrm{O}$ no necesariamente se beneficia de este nivel de PEEP.

\section{PRESIÓN DE DISTENSIÓN}

La presión de distensión o Driving pressure es la diferencia entre la presión meseta y la PEEP (DP = presión meseta - PEEP). Algunos autores se refieren a ella como «el precio de la ventilación» ya que representa la fuerza a «pagar» para obtener cada ventilación y el estrés dinámico aplicado a los pulmones en cada respiración.

Es un reflejo la distensibilidad estática del sistema respiratorio (CRS), como lo sugiere la fórmula necesaria para calcularla: $\mathrm{CRS}=\mathrm{VT} /$ presión de distensión. Donde la presión de distensión es inversamente proporcional a la complianza pulmonar, es decir, ante un volumen tidal dado, un aumento de la PEEP que reclute tejido pulmonar previamente colapsado, la presión de distensión disminuirá y la distensibilidad estática mejorará. Por el contrario, si con un aumento de la PEEP no logra un reclutamiento pulmonar, la presión de distensión aumentará y la distensibilidad estática disminuirá. ${ }^{26}$ Este principio fisiológico de la mecánica respiratoria debe ser utilizado como una herramienta complementaria que permita establecer una PEEP, en la cual se alcanza el reclutamiento pulmonar evitando la sobredistensión.

El análisis retrospectivo de ensayos clínicos del grupo ARDSnet y diversos metaanálisis han mostrado que la presión de distensión es la variable que mejor estratifica el riesgo de muerte en los pacientes con SDRA, con efectos «protectores» de la PEEP sólo cuando ésta se asocia a una menor presión de distensión.

Ajustar el volumen tidal y el nivel de PEEP para obtener una presión de distensión $<15 \mathrm{cmH}_{2} \mathrm{O}$, respetando la estrategia de ventilación protectora pulmonar puede conducir a mejores resultados, especialmente en sujetos con pulmones gravemente lesionados.

Hay que reconocer que el utilizar una estrategia de ventilación protectora pulmonar puede ocasionar hipercapnia, la cual generalmente es bien tolerada sin mayor impacto en el equilibrio ácido-base, se establece el término hipercapnia permisiva como aquélla artificialmente inducida con el objetivo de lograr este objetivo.

No debemos olvidar que la hipercapnia ocasiona mayor crecimiento bacteriano a nivel pulmonar, dificulta la reabsorción de líquido alveolar, inhibe la proliferación de células alveolares y ocasiona hipertensión arterial pulmonar, a la vez que puede ocasionar acidosis e inestabilidad hemodinámica, por tanto, debe tratar de evitarse.

\section{ESTRATEGIAS NO VENTILATORIAS}

\section{Ventilación en posición prona}

La posición prona ofrece grandes beneficios a los sujetos con SDRA por diversos mecanismos como son: la distribución más equitativa de la ventilación, un aumento del volumen pulmonar al final de la espiración, mejora la relación ventilación/perfusión, promueve la eliminación de secreciones, modifica la mecánica de la pared torácica, esto conduce a cambios regionales y mejoras en la ventilación pulmonar global.

La posición prona es un estándar de tratamiento para todos aquéllos que persisten con $\mathrm{PaO}_{2} / \mathrm{FiO}_{2}<150$ con una $\mathrm{FiO}_{2} \geq 60 \%$ y $\mathrm{PEEP} \geq 5 \mathrm{~cm} \mathrm{H} \mathrm{H}_{2}$, tras 12 horas de ventilación mecánica protectora en pacientes con SDRA, según lo publicado por el estudio PROSEVA. ${ }^{27}$ Esto concuerda con las recomendaciones actuales en COVID-19, donde pacientes con SDRA de moderado a grave deben recibir esta terapia.

Está documentada una disminución en la mortalidad de los casos con SDRA que reciben esta forma de tratamiento, con una reducción de hasta $50 \%$ en algunas series, con un bajo número necesario a tratar de 6 . Para que esta estrategia obtenga buenos resultados es importante que se aplique de forma oportuna dentro de las 
primeras 36 horas de iniciada la ventilación mecánica invasiva y mantener por tiempo prolongado, al menos 16 horas según lo publicado por el estudio PROSEVA (promedio $17 \pm 3$ horas) o mayor a 12 horas según lo publicado por metaanálisis subsecuentes.

Es muy importante que ésta se realice por personal calificado con experiencia, para evitar complicaciones incluida la desconexión del ventilador y potencial generación de aerosoles particularmente importante en el caso de COVID-19. Deberá evaluarse de forma objetiva la respuesta al prono, para evitar esfuerzos innecesarios en aquéllos que no son respondedores.

Se considerara respondedor a aquel paciente que presenta un aumento de la $\mathrm{PaO}_{2} / \mathrm{FiO}_{2}$ y/o $\mathrm{PO}_{2}>20 \%$, así como una disminución del $\mathrm{CO}_{2}$ de al menos 0.9 $\mathrm{mmHg}$ con respecto a la posición supina. Se considera un «respondedor rápido» cuando se observan dichos cambios a la hora del cambio de posición y respondedor lento cuando son evidentes hasta las seis horas. ${ }^{28}$ Setenta y cinco por ciento de los pacientes con SDRA son respondedores a la pronación, se desconoce la tasa de respuesta en COVID-19.

Las sesiones de posición prona deben continuar hasta que después de cuatro horas de regresar al paciente a la posición supina, éste mantenga una $\mathrm{PaO}_{2} / \mathrm{FiO}_{2}>$ 150 con $\mathrm{PEEP} \leq 10 \mathrm{cmH}_{2} \mathrm{O}$ y $\mathrm{FiO}_{2} \leq 60 \%$.

\section{Bloqueo neuromuscular}

Recomendar el uso de bloqueo neuromuscular de forma generalizada en casos con SDRA es aún controvertido. En el año 2010 se publicó el ensayo "ACURASYS», ${ }^{29}$ en el que se comparó el uso por 48 horas de una infusión de Besilato de Cisatracurio a $37.5 \mathrm{mg} / \mathrm{hora}$, iniciada en las primeras 48 horas del diagnóstico de SDRA con $\mathrm{PaO}_{2} / \mathrm{FiO}_{2}<150$.

El grupo de intervención tuvo una reducción absoluta de $9 \%$ en la mortalidad a 90 días (31.6 vs $40.7 \%$; $p=$ 0.04; NNT = 11). Reducción en la incidencia de barotrauma ( 5.1 vs $11.7 \% ; p=0.03$ ), neumotórax (4.0 vs $11.7 \% ; p=0.01)$, días fuera de la unidad de cuidados intensivos (47.7 vs. 39.5 días; $p=0.03$ ) y días libres del ventilador (53.1 vs $44.6 \%$; $p=0.03$ ).

Posteriormente, en el año 2019 el grupo de investigación «PETAL" publicó el ensayo «ROSE» 30 el cual asignó al azar a 1,408 adultos con SDRA y relación $\mathrm{PaO}_{2} / \mathrm{FiO}_{2}<150$, a recibir la infusión de besilato de cisatracurio $37.5 \mathrm{mg} /$ hora por 48 horas versus placebo, no encontrando diferencias significativas en la mortalidad a 90 días (42.5 vs $42.8 \%$; $p$ $=0.93$ ). Tampoco en los objetivos secundarios como mortalidad hospitalaria, días libres de ventilación, días libres de la Unidad de Cuidados Intensivos y barotrauma. El grupo de intervención presentó una mayor tasa de debilidad adquirida en la $\mathrm{UCI}$, así como mayor número de eventos adversos y cardiovasculares graves.

Ambos estudios cuentan con una metodología similar pero con resultados totalmente opuestos, por lo cual, actualmente no hay una postura a favor del uso temprano y generalizado del bloqueo neuromuscular. Ya que existe controversia con respecto al beneficio, a la par de que es potencialmente dañina al aumentar el riesgo de debilidad adquirida en la $\mathrm{UCl}$, que en el caso de COVID-19 puede potencializarse con el uso concomitante de corticosteroides.

La evidencia sobre su uso en COVID-19 es limitada y los resultados a largo plazo no están claros. En sujetos con COVID-19 ventilados mecánicamente con SDRA de moderado a grave, las directrices actuales $^{22}$ sugieren el uso de bolos intermitentes de NMBA en lugar de una infusión continua para facilitar la ventilación protectora pulmonar. El uso de infusiones continuas de NMBA durante un máximo de 48 horas debe reservarse para pacientes en los que se necesite optimizar la mecánica pulmonar, por ejemplo, pacientes con presión meseta $>30 \mathrm{cmH}_{2} \mathrm{O}$ y esfuerzos espontáneos vigorosos, o en caso de presentar asincronía grave que no pueda ser corregida con ajustes ventilatorios.

\section{CONCLUSIONES}

La ventilación protectora pulmonar con VT de 6-8 mL/ $\mathrm{kg}$ de peso predicho y presión meseta $<30 \mathrm{cmH}_{2} \mathrm{O}$ ha mejorado los desenlaces clínicos en pacientes con y sin SDRA durante los últimos 20 años, aunque el COVID-19 tiene particularidades que lo caracterizan, la evidencia sugiere continuar con la misma estrategia.

Existen muchos métodos para la titulación de la PEEP, sin embargo, no existe un nivel óptimo, ni una estrategia ideal para su programación. Se recomienda individualizar con base en las características de cada caso, teniendo en mente que alcanzar una «PEEP ideal» es una meta difícil en la práctica médica habitual con recursos técnicos y humanos limitados, por tanto, se debe equilibrar la balanza entre el potencial beneficio vs el daño de cada estrategia implementada.

La heterogeneidad observada en pacientes con COVID-19 ha llevado a diferentes propuestas de manejo con base en los fenotipos descritos. Aunque se sugiere tomar en cuenta estas particularidades en el manejo, no debemos abandonar el tratamiento estándar, la ventilación mecánica protectora pulmonar ha demostrado a lo largo de sus 20 años de aplicación ser una de las pocas medidas que reducen la mortalidad en pacientes con SDRA, intentar cambiar el enfoque de tratamiento sin tener aún evidencia de alta calidad que sugiera hacerlo conlleva potenciales riesgos. 


\section{AGRADECIMIENTOS}

A nuestros profesores, en especial al Dr. Julio César Mijangos Méndez, por el apoyo para la realización de este trabajo.

\section{REFERENCIAS}

1. Kumar M, Taki K, Gahlot R, Sharma A, Dhangar K. A chronicle of SARS-CoV-2: Part-I - epidemiology, diagnosis, prognosis, transmission and treatment. Sci Total Environ. 2020;734(336):139278.

2. Suárez V, Suarez QM. ORS. Epidemiología de COVID-19 en México: del 27 de febrero al 30 de abril de 2020. Rev Clínica Española. 2020;220(8):463-471.

3. Meng Di Jiang ZYZ. Current status of etiology, epidemiology, clinical manifestations and imagings for COVID-19. Korean $J$ Radiol. 2020;

4. Ciotti M, Ciccozzi M, Terrinoni A, Jiang WC, Wang CB, Bernardini S. The COVID-19 pandemic. Crit Rev Clin Lab Sci. 2020;57(6):365-388.

5. Ñamendys-Silva SA, Gutiérrez-Villaseñor A, Romero-González JP. Hospital mortality in mechanically ventilated COVID-19 patients in Mexico. Intensive Care Med. 2020;46(11):2086-2088. doi:10.1007/s00134-020-06256-3.

6. Serpa NA, Oliveira CS, Manetta JA, Galvão MP, Crepaldi ED, de Oliveira Prado Pasqualucci M, et al. Association between use of lung-protective ventilation with lower tidal volumes and clinical outcomes among patients without acute respiratory distress syndrome a meta-analysis. JAMA. 2012;308:1651-1659.

7. ARDS Definition Task Force, Ranieri VM, Rubenfeld GD, Thompson BT, Ferguson ND, Caldwell E, et al. Acute respiratory distress syndrome: the Berlin definition. JAMA. 2012;307:25262533.

8. Marini JJ, Gattinoni L. Management of COVID-19 respiratory distress. JAMA. 2020;323(22):2329-2330.

9. Schenck EJ, Hoffman K, Goyal P, Choi J, Torres L, Rajwani K, Tam CW, et al. Respiratory mechanics and gas exchange in COVID-19-associated respiratory failure. Ann Am Thorac Soc. 2020;17(9):1158-1161.

10. Cummings MJ, Baldwin MR, Abrams D, Jacobson SD, Meyer BJ, Balough EM. Epidemiology, clinical course, and outcomes of critically ill adults with COVID-19 in New York City: a prospective cohort study. Lancet. 2020;395(10239):1763-1770.

11. Calfee CS, Delucchi K, Parsons PE, Thompson BT, Ware LB MMNAN. Subphenotypes in acute respiratory distress syndrome: latent class analysis of data from two randomised controlled trials. Lancet Respir Med. 2014;2(8):611-620.

12. Brower RG, Matthay MA, Morris A, Schoenfeld D, Taylor TB, Wheeler A. Ventilation with lower tidal volumes as compared with traditional tidal volumes for acute lung injury and the acute respiratory distress syndrome. N Engl J Med. 2000;342(18):13011308.

13. Lellouche F, Dionne S, Simard S, Bussières JS, Dagenais F. High tidal volumes in mechanically ventilated patients increase organ dysfunction after cardiac surgery. Anesthesiology. 2012;116(5):1072-1082.

14. Futier E, Constantin JM, Paugam-Burtz C, Pascal J, Eurin $\mathrm{M}$, Neuschwander A, et al. A trial of intraoperative lowtidalvolume ventilation in abdominal surgery. N Engl J Med 2013. 2013;369:428-437.

15. Brower RG, Lanken PN, Maclntyre N, Matthay MA, Morris A, Ancukiewicz M, et al. Higer versus lower positive end expiratory pressure in patients with acute respiratory distress syndrome. $N$ Engl J Med. 2004;351(4):327-36.

16. Mercat A, Richard JC, Vielle B, Jaber S, Osman D, Diehl JL, et al. Positive end-expiratory pressure setting in adults with acute lung injury and acute respiratory distress syndrome: a randomized controlled trial. JAMA. 2008;299(6):646-655.

17. Kacmarek RM, Villar J, Sulemanji D, Montiel R, Ferrando C, Blanco J, et al. Open lung approach for the acute respiratory distress syndrome: a pilot, randomized controlled trial. Crit Care Med. 2016;44(1):32-34.

18. Writing Group for the Alveolar Recruitment for Acute Respiratory Distress Syndrome Trial (ART) Investigators, Cavalcanti AB, Suzumura EA et al. Effect of lung recruitment and titrated positive end-expiratory pressure (PEEP) vs low PEEP on mortality in patients with acute respiratory distress syndrome: a randomized clinical trial. JAMA. 2017;318(14):1335-1345.

19. Talmor D, Sarge T, Malhotra A, O'Donnell CR, Ritz R, Lisbon A, et al. Mechanical ventilation guided by esophageal pressure in acute lung injury. N Engl J Med. 2008;359(20):2095-2104.

20. Beitler JR, Sarge T, Banner-Goodspeed VM, Gong MN, Cook $D$, Novack V, et al. Effect of titrating positive end-expiratory pressure (PEEP) with an esophageal pressure-guided strategy vs an empirical high PEEP-FiO, strategy on death and days free from mechanical ventilation among patients with acute respiratory distress syndrome: A R. JAMA. 2019;321(9):846-857.

21. Poston JT, Patel BK DA. Management of critically ill adults with COVID-19. JAMA. 2020;323(18):1839-1841.

22. Alhazzani W, Møller MH, Arabi YM, Loeb M, Gong MN, Fan E et al. Surviving sepsis campaign: guidelines on the management of critically ill adults with coronavirus disease 2019 (COVID-19). Intensive Care Med. 2020;46(5):854-887.

23. Lentz S, Roginski MA, Montrief T, Ramzy M, Gottlieb M, Long B. Initial emergency department mechanical ventilation strategies for COVID-19 hypoxemic respiratory failure and ARDS. Am J Emerg Med. 2020;38(10):2194-2202.

24. Pan C, Chen L, Lu C, Zhang W, Xia JA, Sklar MC et al. Recruitability in COVID-19-associated acute respiratory distress syndrome: a single-center observational study. Am J Respir Crit Care Med. 2020;201(10):1294-1297.

25. Chiumello D, Cressoni M, Carlesso E, Caspani L, Marino A, Gallazzi E. Bedside selection of positive end-expiratory pressure in mild, moderate, and severe acute respiratory distress syndrome. Crit Care Med. 2014;42(2):252-264.

26. Aoyama H, Yamada Y, Fan E. The future of driving pressure: a primary goal for mechanical ventilation? J Intensive Care. 2018;6(64).

27. Guérin C, Reignier J, Richard JC, Beuret P, Gacouin A, Boulain $\mathrm{T}$, et al. Prone positioning in severe acute respiratory distress syndrome. N Engl J Med. 2013;368(23):2159-2168.

28. Papazian L, Paladini MH, Bregeon F, Huiart L, Thirion X, Saux $P$, et al. Is a short trial of prone positioning sufficient to predict the improvement in oxygenation in patients with acute respiratory distress syndrome? Intensive Care Med. 2001;27(6):1044-1049.

29. Papazian L, Forel JM, Gacouin A, Penot-Ragon C, Perrin G, Loundou $A$, et al. Neuromuscular blockers in early acute respiratory distress syndrome. N Engl J Med. 2010;363(12):1107-1116.

30. National Heart, Lung and BIPCTN. Early neuromuscular blockade in the acute respiratory distress syndrome. $N$ Engl $J$ Med. 2019;380(21):1997-2008.

Patrocinios: Ninguno.

Relación de conflicto de intereses: Ninguno.

\section{Correspondencia:}

Dra. Yessica García Salas

Hospital Civil de Guadalajara «Fray Antonio Alcalde»,

Calle Hospital Núm. 278,

Col. El Retiro, 44280,

Guadalajara, Jalisco, México.

Tel: 3339424400.

E-mail: dest_yessi@hotmail.com 